\title{
Improved Access to Healthcare is Good for Everyone
}

\author{
Charles R. Scoggins, MD, MBA, and Michael E. Egger, MD, MPH \\ Department of Surgery, Division of Surgical Oncology, University of Louisville, Louisville, KY
}

As a community, we strive to make the lives of our patients better. There are enormous research efforts in drug development, outcomes research, and population health. Combine these investigative activities with continuous improvements in clinical care and you have a recipe for quality healthcare that reaches across all aspects of the care delivery continuum. However, for an individual patient to enjoy this improved healthcare, they (usually) need some form of health care funding in the form of insurance (be it private insurance or governmental). Unfortunately, there are too many uninsured people in the US. In 2010, the Affordable Care Act (ACA) attempted to narrow the gap between the insured and uninsured by creating Medicaid Expansion (ME). This resulted in nearly 20 million lowincome people obtaining health coverage via federally supported expansion of Medicaid eligibility on a state-bystate basis. With all these newly funded patients, questions began to arise about the impact of ME on a variety of disease and health processes. Subsequently, the National Cancer Database (NCDB) added a variable in the 2016 Patient Use Data File (PUF), released 2 years ago, denoting whether a patient lived in an ME state or non-ME state. This has allowed for research focused on the effect of expanded health coverage and its association with outcomes for cancer care, including better access to the processes of care.

How do we make cancer care better? Some strategies include developing better, less toxic treatments, improving screening for earlier stages of disease, and enhancing access to care. It seems intuitive that reducing the number

(C) Society of Surgical Oncology 2021

First Received: 19 August 2021

Accepted: 31 August 2021;

Published Online: 17 September 2021

C. R. Scoggins, MD, MBA

e-mail: charles.scoggins@louisville.edu of uninsured people might increase utilization of medical services by reducing the financial barrier. This, of course, is not a one-to-one relationship, but by providing health coverage for those who have none, we presumably improve affordability of care, thus increasing health care utilization. The Oregon health insurance experiment demonstrated that ME increased health care utilization, as assessed by emergency department use. ${ }^{1}$ But what about utilization of surgical care? Work by Al-Refaie et al., using pre-ACA administrative data, would suggest yes. ${ }^{2}$ Enhancing access could result in more screening for those cancers where an effective screening program exists (examples include breast and colon cancer). We have seen the impact of expanded healthcare access on cancer screening and the news is good: patients who were previously disadvantaged are now having access to screening mammograms and colonoscopies. ${ }^{3,4}$ This has resulted in a shift towards earlier stages of disease with the expected better survival. ${ }^{3,4}$

But what about cancers that lack meaningful screening programs? Would expanded access to care (and the various processes of oncologic care) have an impact on patients' lives? In this issue, Fonseca and colleagues looked at data from the NCDB and sought to address this very simple question: what is the impact of ME on pancreatic cancer treatment and outcomes? ${ }^{5}$ What they found is important; those patients who lived in states with ME had better utilization of the various processes of care that most experts associate with quality pancreatic cancer care. Patients from ME states were more likely to receive multimodal therapy than those from non-ME states (odds ratio [OR] 1.6). This includes multiagent chemotherapy (OR 1.49), neoadjuvant chemotherapy (OR 1.39), and undergoing resection of their cancer (OR 1.54). These data are compelling and clearly demonstrate the beneficial effect that policy has on healthcare delivery, especially complex oncologic therapy. Furthermore, patients from ME states were more likely to be treated at high-volume centers (OR 1.57). In summary, patients with pancreas cancer in ME states were $50 \%$ more 
likely to check all the boxes that we associate with highquality multidisciplinary care compared with otherwise similar pancreas cancer patients in non-ME states.

The benefits of expanded healthcare access were not limited to the processes of care. The authors also reported better oncologic outcomes for patients who lived in $\mathrm{ME}$ states. Not only were perioperative measures of quality better (fewer postoperative deaths, which might be a highvolume center effect) but the overall survival was longer for patients from ME states (hazard ratio 0.82). ${ }^{5}$ Few things are more compelling in oncology than improved overall survival. This is direct evidence that national policy directly improved survival for a disease that lacks a meaningful screening tool. In other words, there was not a stage migration seen with expanded access, but instead better cancer care.

So how did ME improve these measures for patients across the board, and not just patients with Medicaid? It is important to note that the median age of the cohort studied in the article by Fonseca et al. is 66 years. ${ }^{5}$ This means that half of the patients in the study were covered by Medicare, therefore ME should not impact the affordability of medical care for these patients, yet the effect is strong across all age groups. What is going on? It is important to remember that the ACA did not just simply hand an insurance card to more patients in poverty in states that chose to accept federally subsidized Medicaid eligibility expansion. The ACA subsidized employer-sponsored health insurance coverage and increased funding to community health centers, yet these subsidies were applied nationally, regardless of state-level ME. Presumably, some of these other aspects of the ACA would be improving access to care for other underserved patients, not just Medicaid-eligible patients. What is so interesting about the findings of the paper by Fonseca et al. is that ME in and of itself seemed to allow all boats to rise for all residents in ME states compared with non-ME states. ${ }^{5}$ ME expansions may be improving the overall effectiveness of the care delivery system for Medicaid and non-Medicaid residents. More research is needed to evaluate these tantalizing findings further.

There are some additional analyses that we wish Fonseca et al. could have provided to help further explore these issues. Subgroup analyses for rural patients, urban poor patients, Medicaid-covered patients, and non-White patients would have been of interest to see where ME has had the greatest or least effect. These findings would tell us where the rate-limiting steps of the care delivery continuum exist after ME. We wish an analysis on a state-bystate level could be performed, in which delivery of highquality, multimodality care for pancreas cancer could be assessed in a before and after analysis on the state level.
However, one limitation of the NCDB is that state of residence is not provided, not even in a blinded fashion; only nine different geographic regions are provided.

It should be discussed that several other improvements occurred around the same time period that was studied by Fonseca and colleagues. First, as correctly pointed out by the authors, the concept of multi-agent chemotherapy was shown to be better than single-agent chemotherapy for pancreas cancer. The authors nicely demonstrated that patients were $60 \%$ more likely to receive multi-agent chemotherapy if they lived in a Medicaid state. This seems to be associated with improved access to quality oncologic care. Furthermore, FOLFIRINOX has been shown to be the most effective regimen to date. ${ }^{6}$ Although this level of granularity cannot be gleaned from the NCDB, a proxy for this would be multiagent chemotherapy.

We are getting better at treating pancreas cancer. Those of us who care for these patients every day are convinced that patients are best treated in high-volume, multidisciplinary settings. Multi-agent chemotherapy, liberal use of neoadjuvant therapy, and treatment in high-volume centers are likely some the reasons for the improvement in survival we are seeing for patients with localized pancreas cancers. The findings in this paper suggest that recent government initiatives to improve health care delivery in this country are improving access to high-quality multimodality therapy for pancreas cancer. The next step is for these high-volume centers to evaluate their own processes of care delivery and ask the question, "Are we doing everything we can to improve access to care at our own facility for underserved patients?"

DISCLOSURES Charles R. Scoggins and Michael E. Egger declare no conflicts of interest.

\section{REFERENCES}

1. Taubman SL, Allen HL, Wright BJ, Baicker K, Finkelstein AN. Medicaid increases emergency-department use: evidence from Oregon's health insurance experiment. Science. 2014;343:263-8.

2. Al-Refaie WB, Zheng C, Jindal M, Clements ML, Toye P, Johnson LB, et al. Did pre-affordable care act medicaid expansion increase access to surgical cancer care? $J$ Am Coll Surg. 2017;224(4):662-9.

3. Gan T, Skinner HF, Walling SC, Chen Q, Huang B, Tucker TC, et al. Impact of the affordable care act on colorectal cancer screening, incidence, and survival in kentucky. J Am Coll Surg. 2019;228(4):342-52.

4. Ajkay N, Bhutiani N, Huang B, Chen Q, Howard JD, Tucker TC, et al. Early impact of medicaid expansion and quality of breast cancer care in kentucky. $J$ Am Coll Surg. 2018;226(4):498-506.

5. Fonseca AL, Cherla D, Kothari AN, Tzeng CWD, Heslin MJ, Mehari KR, et al. Association of Medicaid expansion with pancreatic cancer treatment and outcomes: evidence from the National Cancer Database. Ann Surg Oncol. 2021. https://doi.org/ 10.1245/s10434-021-10709-4. 
6. Conroy T, Desseigne F, Ychou M, Bouche O, Guimbaud R, Becouarn Y, et al. FOLFIRINOX versus gemcitabine for metastatic pancreatic cancer. $N$ Engl J Med. 2011;364(19):

Publisher's Note Springer Nature remains neutral with regard to $1817-25$. jurisdictional claims in published maps and institutional affiliations. 